\title{
Credit-Based Fair Queueing (CBFQ):A simple and feasible scheduling algorithm for packet networks
}

\author{
B. Bensaou, K. T. Chan, and D. H. K. Tsang \\ Department of Electrical \& Electronic Engineering \\ Hong Kong University of Science \& Technology \\ Clear Water Bay, Kowloon, Hong Kong \\ E-mail:\{eebben, eeking, eetsang\}@ee.ust.hk \\ Tel: +852 23587080 Fax: +8522358 1485
}

\begin{abstract}
A simple rate-based scheduling algorithm for packet-switched networks (e.g. Internet, ATM) is proposed. By using simple counters to trace the amount of credits accumulated by each traffic stream, the algorithm decides which stream to serve next based on the bandwidth share allocated to each traffic stream and the sizes of their respective head of line (HOL) packets. Our proposed CBFQ algorithm achieves the same fairness and delay bounds as the alternative approaches based on virtual time, while avoiding the implementation problems inherent in these methods. In addition, to take advantage of the fixed packet size in ATM networks, a variant of the algorithm (CBFQ-F) is proposed. By relaxing the fairness bound by a negligible amount, CBFQ-F achieves a complexity of $O(1)$.

Keywords: Fair queueing, Bandwidth scheduling, Delay bound, Packet-switched networks.
\end{abstract}

\section{Introduction}

To support both real-time and non real-time applications, the network needs to provide guarantee of the end-to-end quality of service (QoS). This requires the network to provide in each multiplexer enough buffers to avoid packet loss and to use the necessary scheduling algorithms to meet the packet delay requirements. To achieve this latter issue, fair queueing scheduling algorithms proved very efficient. In this spirit, many algorithms such as packet generalized processor sharing (PGPS) [1], self clocked fair queueing (SCFQ) [2] have been proposed. Mostly all the algorithms are based on the idea of associating to each arriving packet a virtual time stamp calculated with respect to an increasing reference virtual clock. The smaller the time stamp, the higher is the priority of the corresponding packet to access the transmission facilities. These algorithms proved very efficient in guaranteeing the fairness among the traffic streams. However, the inherent complexity of the virtual time approach makes the practical implementation very difficult and/or expensive. Moreover, the use of a reference clock implies that it cannot be reset to zero until the system is idle. Unless the implementation of this kind of algorithm oversizes drastically the registers holding the value of the virtual clock, this can induce a potential problem of numerical overflow, when the system remains busy for long periods. In this case, the virtual clock wraps a round and lead to the unstability of the algorithm. In the following, we propose an algorithm which uses simple counters to keep track of the credits accumulated by each traffic stream (or QoS class). Based on the values of the counters, the sizes of the head-of-line (HOL) packets of the different streams, and the share of bandwidth allocated to the different traffic streams, the algorithm decides which packet is to be sent next. In our algorithm, we can proof that the values of the counters are bounded by the size of the longest packet, which avoids any numerical overflow problem.

\section{CBFQ: the general algorithm}

\subsection{The network node model}




\subsection{Performance of CBFQ}

Fairness bound: Let $\mathcal{J}\left(t_{1}, t_{2}\right)$ denote the set of queues that are continuously backlogged during $\left(t_{1}, t_{2}\right)$. Define $W_{i}\left(t_{1}, t_{2}\right)$ and $M A X_{i}, i=1, \ldots, J$, as the amount of traffic transmitted from queue $i$ during $\left(t_{1}, t_{2}\right)$ and the maximum packet size of stream $i$ respectively. We can prove the following fairness bound.

$$
\forall l, l^{\prime} \in \mathcal{J}\left(t_{1}, t_{2}\right),\left|\frac{W_{l}\left(t_{1}, t_{2}\right)}{S_{l}}-\frac{W_{l^{\prime}}\left(t_{1}, t_{2}\right)}{S_{l^{\prime}}}\right| \leq \frac{M A X_{l}}{S_{l}}+\frac{M A X_{l^{\prime}}}{S_{l^{\prime}}} .
$$

The fairness bound guaranteed by CBFQ is as expected the same as the ones guaranteed by the alternative algorithms such as SCFQ and PGPS. This statement can be proved by, first, proving that $0 \leq K_{l} \leq M A X_{l}$, at any time $t$; and second, if we let $A_{l}\left(t_{1}, t_{2}\right)$ be the amount of credit earned by stream $l$ during $\left(t_{1}, t_{2}\right)$, we can show that $W_{l}\left(t_{1}, t_{2}\right) \leq A_{l}\left(t_{1}, t_{2}\right)+K_{l}\left(t_{1}\right)-K_{l}\left(t_{2}\right)$. Finally, by writing explicitly the expression for $A_{l}\left(t_{1}, t_{2}\right)$, the proof of the bound becomes straightforward.

End-to-end delay bound: Denote by $d_{l}^{n}$ the end-to-end delay experienced by the $n$th packet from stream $l$. Let $I$ be the number of nodes on the path of flow $l$, and $C_{i}, i=1, \cdots, I$ be the server capacity at node $i$. Define $\mathcal{N}_{i}$ as the set of streams served by node $i$. Let $M A X_{l}$ be the maximum packet size from stream $l$, and $M A X_{l}^{n}$ be the maximum packet size from stream $l$ up to packet $n$ (i.e. $M A X_{l}^{n}=\max _{k \in\{1, \ldots, n\}} L_{l}^{k}$, where $L_{l}^{k}$ is the length of the $k^{t h}$ packet from stream $l$ ).

For a stream $l$, constrained by a $\left(\sigma_{l}, \rho_{l}\right)$-Leaky Bucket, if the scheduling algorithm at each node on the path of the flow is CBFQ then, using the framework provided in [3], we can prove that the end-to-end delay bound for packet $n$ of stream $l$ is given by

$$
d_{l}^{n} \leq \frac{\sigma_{l}+(I-1) M A X_{l}^{n}}{\rho_{l}}+\sum_{i=1}^{I} \sum_{m \in \mathcal{N}_{i} \backslash\{l\}} \frac{M A X_{m}}{C_{i}}+\sum_{i=1}^{I-1} \tau_{i},
$$

where $\tau_{i}$ is the propagation delay between nodes $i$ and $i+1$. This delay bound proved to be the same as the one guaranteed by the SCFQ algorithm.

Complexity of CBFQ: It is easy to prove that after serving a packet, the order of the terms in Step 4 of the algorithm above does not change, except for the queue that has just been served. In this case it is not required to fully sort the queues as in Step 4 each time a packet is served. This operation reduces rather to the insertion of an element (either the queue that has just been served, or a queue that just became active) in an already fully sorted list. Besides, since the counters are independent, the update operation in Step 5 can take place in parallel making the complexity of this step $O(1)$. With these remarks and knowing all the other operations are $O(1)$, the complexity of the algorithm becomes the same as the complexity of a search algorithm: $O(\log (J))$. Note that PGPS is not feasible practically since it relies on the real-time simulation of a fluid fair queueing system to maintain the virtual clock. The SCFQ algorithm, on the other hand, as presented originally by Golestani [4], requires $O(\log (n))$ where $n$ is the number of packets in the system. More recently, new implementations of SCFQ algorithm has shown a complexity of $O(\log (J))[5,6]$, however, this is achieved at the expense of more complexe hardware devices. By comparison to the alternative approaches, CBFQ achieves major improvements towards practical implementations.

\section{CBFQ-F: A version for fixed packet size networks}

In CBFQ, the decision process to choose the next packet to serve requires operations as complex as $O(\log (J))$, for each packet. When the packets lenghts are relatively long, as it is the case in Internet, this processing overhead is acceptable. In an ATM network environment, where the packets are short 
downgrade implicitly the effective throuthput of the link to less than $50 \%$. To satisfy the requirements in processing speed in ATM networks, CBFQ-F sacrifices a small amount on the fairness bound to achieve smaller processing overheads as shown below.

\subsection{CBFQ-F algorithm}

The CBFQ-F algorithm, is based on the set of counters $K_{i}, i=1, \cdots, J$, associated with each queue. Frames (or cycles of service) of variable length are defined without any prior knowledge of their length. At the beginning of each cycle, the backlogged queve $j_{1}$ whose share of bandwidth $S_{j_{1}}$ is the largest among the active queues is chosen as the reference queue to calculate the relative share of bandwidth each backlogged queue should receive in the cycle. All the counters are then increased by this relative share which is simply the ratio of the queue's share to $S_{j_{1}}$. The queues whose counters reach the value of one should receive one packet-time service. Following this idea, first, there will be no wasted (empty) time slot as long as there is at least one active queue, since each time at least the counter of queue $j_{1}$ is equal to one; and second, all the active streams receive bandwidth proportionally to their shares. Accordingly, the algorithm operates as follows:

1. Initialize: At the beginning of operation, set all the counters to zero: $K_{l}=0, \quad l=1, \cdots, J$.

2. Begin: (cycle) Let $\mathcal{J}=\left\{j_{1}, \cdots, j_{k}\right\}$ and $|\mathcal{J}|=k$ such that Queues $j_{1}, \cdots, j_{k}$ are currently backlogged and $S_{j_{1}} \geq S_{j_{2}} \geq \cdots \geq S_{j_{k}}$

3. Update the counters as follows:

$$
K_{j_{n}} \longleftarrow K_{j_{n}}+\frac{S_{j_{n}}}{S_{j_{1}}}, \quad \forall j_{n} \in \mathcal{J}
$$

4. Let $\mathcal{K}=\left\{\ell \in \mathcal{J}: K_{\ell} \geq 1\right\}$

5. Loop (while $\mathcal{K}$ is non-empty)

Serve one packet (or cell) from queue $\ell \in \mathcal{K}$ and update its counter by

$$
K_{\ell} \longleftarrow\left\{\begin{array}{cl}
K_{\ell}-1 & \text { if queue } \ell \text { is non-empty; } \\
0 & \text { if queue } \ell \text { is empty. }
\end{array}\right.
$$

$\mathcal{K} \longleftarrow \mathcal{K} \backslash\{\ell\}$

\section{End Loop}

6. End (cycle).

\subsection{Performance of CBFQ-F}

Fairness guarantee: Consider the interval of time $\left(t_{1}, t_{2}\right)$ and define $j_{1}\left(t_{1}\right)$ and $j_{1}\left(t_{2}\right)$ as the reference queues at times $t_{1}$ and $t_{2}$ respectively. With the same definitions as for CBFQ, we can prove the following fairness bound for CBFQ-F

$$
\forall t_{1}, t_{2} \text { and } \forall l, l^{\prime} \in \mathcal{J}\left(t_{1}, t_{2}\right),\left|\frac{W_{l}\left(t_{1}, t_{2}\right)}{S_{l}}-\frac{W_{l^{\prime}}\left(t_{1}, t_{2}\right)}{S_{l^{\prime}}}\right| \leq\left(\frac{1}{S_{l}}+\frac{1}{S_{l^{\prime}}}+\frac{2}{S_{j_{1}}^{*}}\right) L
$$

where $S_{j_{1}}^{*} \triangleq \min \left(S_{j_{1}\left(t_{1}\right)}, S_{j_{1}\left(t_{2}\right)}\right)$. The fairness bound guaranteed by CBFQ-F is, as expected, slightly larger than the ones guaranteed by the alternative algorithms such as SCFQ, PGPS and Virtual Spacing (VS) [5]. However, compared to the bound guaranteed by the deficit round robin (DRR) algorithm [7] which proved to require the same complexity as CBFQ-F, it proved to be orders of 
End-to-end delay bound guarantee: Let $I$ be the number of nodes on the path of stream $l$. Define $N_{i}, i=1, \cdots, I$ as the number of streams flowing through node $i$. Denote by $C_{i}$ the output capacity of node $i$, and $\tau_{i}$ the propagation delay between nodes $i$ and $i+1$. Define $S_{j(., i)}$ as the share of the $j$ th flow in node $i$ and $\hat{S}_{i} \equiv \min _{j} S_{j}(., i)$ as the smallest share among the streams flowing through node $i$. For a stream $l$ which is constrained by a $\left(\sigma_{l}, \rho_{l}\right)$-Leaky Bucket, if the scheduling algorithm at each server on the path of the flow is CBFQ-F, then using the framework provided in [3], we can prove that the end-to-end delay bound for packet $n$ of stream $l$ is given by

$$
d_{l}^{n} \leq \frac{\sigma_{l}+(I-1)}{\rho_{l}}+\sum_{i=1}^{I}\left(\frac{N_{i}-1}{C_{i}}+\frac{2}{\hat{S}_{i}}+\tau_{i}\right)
$$

This delay bound is slightly larger than the ones provided by SCFQ or VS algorithms.

Complexity of CBFQ: The bandwidth shares are allocated at the connexion setup and remain constant for the lifetime of the connexion. The only changes in the priority order in Step 2 of the algorithm occurs thus in a time scale much larger than the packet level time-scale. In particular, unless a connexion departs or a new one arrives, the order does not change. In this case, it is reasonable to assume that at the packet-level time scale, the complexity of Step 2 is $O(1)$. In Step 4 of the algorithm, the elements of the set $\mathcal{K}$ are not sorted according to their counters values, which means, the construction of $\mathcal{K}$ and the selection of the elements of $\mathcal{K}$ who will receive service next is done only by comparing the values of their counters to 1 . This can be practically implemented by very simple hardware devices such as gates, performing only $O(1)$ operations. All the other operations are $O(1)$. Thus, each time a packet is selected for service, an $O(1)$ computational overhead is required. By comparison to the alternative approaches such as SCFQ, PGPS and VS, this constitutes a major improvement towards practical implementations. On the other hand, compared to the DRR algorithm, CBFQ-F achieves the same complexity while guaranteeing much more attractive fairness and delay bounds.

\section{Conclusion}

A new scheduling algorithm CBFQ for packet-switched networks has been proposed. Based on a set of counters that keep track of the credits earned by each traffic stream, CBFQ decides which stream is to be served next. The CBFQ algorithm achieves the same fairness and delay bounds as the alternative algorithms such as SCFQ while requiring less computational overhead or hardware for practical implementation. To reduce the complexity further, CBFQ-F, a version for fixed packet size networks has been proposed. CBFQ-F reduces substantially the computational overhead, however, this is achieved at the expense of a slightly more relaxed fairness bound.

\section{References}

[1] A. K. Parekh and R. G. Gallager, "A generalized processor sharing approach to flow control in integrated services network," in Proceedings of the IEEE INFOCOM, pp. 915-924, 1992.

[2] S. J. Golestani, "A self-clocked fair queueing scheme for broadband applications," in Proceedings of the IEEE INFOCOM, pp. 636-646, 1994.

[3] P. Goyal, S. Lam, and H. Vin, "Determining end-to-end delay bounds in heterogeneous networks," in Proc. 5th Int. Workshop on Network and Operating System Support For Digital Audio and Video, pp. 287-298, April 1995.

[4] S. J. Golestani, "Fair queueing algorithms for packet scheduling in BISDN," in LNCS 1044: Broadband Communications, 1996 International Zurich Seminar on Digital Communications., pp. 39-51, Springer, 1996. 
[6] J. L. Rexford, A. G. Greenberg, and F. G. Bonomi, "Hardware-efficient fair queueing architectures for high-speed networks," in IEEE INFOCOM'96, pp. 638-646, 1996.

[7] M. Shreedhar and G. Varghese, "Efficient fair queueing using deficit round-robin," IEEE/ACM Transactions on Networking, vol. 4, pp. 375-385, June 1996. 